Gynäkologe 2012 · 45:9-10

DOI 10.1007/s00129-011-2855-y

Online publiziert: 15. Januar 2012

(c) Springer-Verlag 2012

W. Jonat ${ }^{1} \cdot$ W. Distler ${ }^{2} \cdot \mathrm{K}$. Diedrich ${ }^{3} \cdot$ N. Arnold ${ }^{1}$

${ }^{1}$ Klinik für Gynäkologie und Geburtshilfe, Universität Schleswig-Holstein, Campus Kiel

${ }^{2}$ Klinik und Poliklinik für Frauenheilkunde und Geburtshilfe, Universitätsklinikum Carl Gustav Carus Dresden

${ }^{3}$ Klinik für Frauenheilkunde und Geburtshilfe, Universitätsklinikum Schleswig-Holstein, Campus Lübeck

\title{
Technologische Weiterentwicklung als Basis für eine personalisierte Medizin
}

gen. Die Übersichtsarbeit von Zapatka und Lichter erläutert Ihnen die Prinzipien dieser Verfahren und beleuchtet auch das diagnostische Potenzial, das in diesen Verfahren liegt.

Neben dem technologischen Fortschritt spielen, wie in der Arbeit von Frau Nöthlings dargelegt wird, Biobanken eine große Bedeutung für die Forschung im Zeitalter der personalisierten Medizin. Gut geführte Biobanken sind ein Garant, dass die darin enthaltenen Biomaterialien nach definierten Standards gesammelt und prozessiert werden. Damit eine Biobank erfolgreich entwickelt werden kann, erfordert sie die Bereitschaft der Ärzte, also auch von Ihnen, zur entsprechenden Aufklärung der Patienten und zur Entnahme der Biomaterialien.

Es wurde natürlich schon immer versucht, die Behandlung nach Diagnosestellung an jeden einzelnen Patienten anzupassen. Mit den neuen Techniken in der Genom- und Transkriptomanalyse eröffnen sich neben den traditionellen Ansätzen zum Verständnis und zur Behandlung von Krankheiten durch Einbeziehung von molekularen Marken vollkommen neue Optionen für prädiktive und prognostische Aussagen. Dieser Herausforderung hat sich, wie Frau Lebeau in ihrem Beitrag darlegt, die Pathologie gestellt. Doch trotz des Fortschritts auf der präklinischen Forschungsebene ist der Transfer dieser Er- kenntnisse in den klinischen Alltag immer noch gering und verläuft sehr langsam.

Für Gynäkologen sind derzeit zwei Tumorsyndrome von besonderer Bedeutung: das familiäre Brust-und-Eierstockkrebs-Syndrom („hereditary breast ovarian cancer syndrome", HBOC) und das familiäre Kolonkarzinom-ohne-PolyposisSyndrom („hereditary non-polyposis colorectal carcinoma syndrome“, HNPCC, Lynch-Syndrom). Diese werden in dem Beitrag von Kast und Arnold abgehandelt. Aufgrund der im Vergleich zum Mammakarzinom niedrigeren Inzidenz von Endometriumkarzinomen rückt die klinische Relevanz des HNPCC-Syndroms erst allmählich in das Bewusstsein der betreuenden Ärzte.

Wie Weichert und Eckmann darlegen, hat sich in den letzten 20 Jahren ein Wandel weg von der invasiven Pränataldiagnostik hin zur nichtinvasiven Ultraschalltechnik mit Ersttrimesterscreening und entsprechender Risikokalkulation für Aneuploidien sowie dem Zweittrimesterscreening vollzogen. Dieser Wandel beruht nicht zuletzt darauf, dass, wie sie in ihrer Arbeit darlegen, in den letzten Jahren neue, risikoarme und verlässliche Methoden sowohl für ein effizientes pränatales Screening als auch für die pränatale Diagnostik genetischer Störungen entwickelt wurden. Dies führt dazu, dass chung der kompletten humanen Sequenz die sogenannte postgenomische Ära prä- 
die Zahl der invasiven pränataldiagnostischen Eingriffe kontinuierlich abnimmt.

\section{》) Dank neuer pränataldiag- nostischer Verfahren nimmt die Zahl der invasiven Eingriffe $a b$}

Der Problembereich, den das Gendiagnostikgesetz (GenDG) in diesem Umfeld schafft, wird in dem Referat ebenfalls angesprochen. Im GenDG ist für diese Art von Untersuchungen eine Aufklärung bzw. eine humangenetische Beratung vorgeschrieben. Durch die Anzahl der in Deutschland im Jahr durchgeführten Untersuchungen, wie von Weichert und Eckmann erwähnt: 500.000, gestaltet sich die praktische Umsetzung des GenDG schon allein durch den momentanen Mangel an humangenetisch ausgebildeten Fachpersonal als schwierig.

Im Beitrag von Diedrich et al. wird ein Thema beschrieben, das vor allem in den letzten Jahren, durch die beschrieben neuen technologischen Möglichkeiten, und insbesondere in letzter Zeit mit der Diskussion um die Gesetzesvorlage in der Gesellschaft recht kontrovers diskutiert wurde. Mit der Entscheidung des Bundestages vom 07.06.2011 ist die Präimplantationsdiagnostik unter strengen Voraussetzungen auch in Deutschland rechtlich möglich geworden. Hintergründe zu dem Gesetzesverfahren werden in dem Artikel ausführlich dargestellt. Abgerundet wird die Arbeit durch einen eigenen Fall, der die Vorteile der PID eindrucksvoll belegt.

Auch wenn die Themen in dem Beitrag, insbesondere die Grundlagen betreffend, recht komplex sind, so hoffen wir doch, dass sie mit dieser Ausgabe von Der Gynäkologe einen Einblick in die neuen technologischen Möglichkeiten und ihre heutigen wie - insbesondere - künftigen Anwendung in der Frauenheilkunde erhielten.

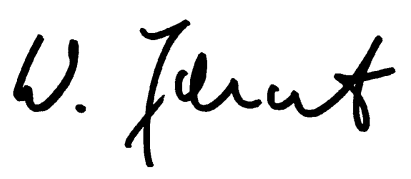

Prof. Dr. Walter Jonat

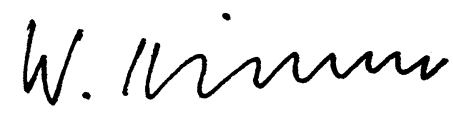

Prof. Dr. Wolfgang Distler

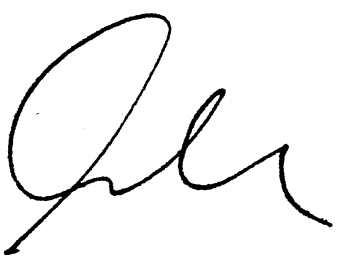

Prof. Dr. Klaus Diedrich

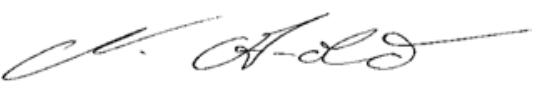

Prof. Dr. Norbert Arnold

\section{Korrespondenzadresse}

Prof. Dr. W. Jonat

Klinik für Gynäkologie und Geburtshilfe, Universität Schleswig-Holstein, Campus Kiel 24105 Kiel

jonat@email.uni-kiel.de

\section{Internistische Erkrankungen in der Schwangerschaft}

Heutzutage sind 2 von 3 Schwangerschaften als Risikoschwangerschaft einzuschätzen. Diese deutliche Zunahme innerhalb von 2 Jahrzehnten lässt sich neben dem Altersanstieg der schwangeren Frauen auch durch die aktive Erhebung von Risikokonstellationen und vermehrte Vorsorge erklären. Maßnahmen wie die hochauflösende Ultraschalluntersuchung verbessern die antenatale Aufdeckung auch geringer Fehlbildungen, die automatisch zum Status einer Risikoschwangerschaft führen. So reflektieren diese Zahlen insbesondere auch die erfolgreichen Bemühungen um

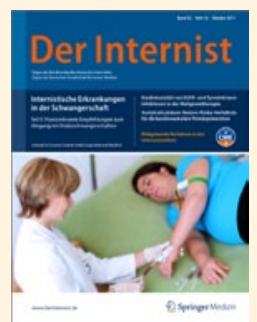
eine Intensivierung der Risikovorsorge. Das Thema „Risikoschwangerschaften" behandelt die Zeitschrift Der Internist in Heft 10/2011.

Die Schwerpunkte des Leitthemenheftes sind:

- Gestationsdiabetes

- Schilddrüsenerkrankungen und Schwangerschaft

- Schwangerschaft und Nierenerkrankungen

- Schwangerschaft unter Immunsuppression

- Folgen von Alkohol und Rauchen in der Schwangerschaft

Bestellen Sie diese Ausgabe zum Preis von $34 €$ bei

Springer Customer Service Center Kundenservice Zeitschriften Haberstr. 7 69126 Heidelberg Tel.: +49 6221-345-4303 Fax: +49 6221-345-4229

E-Mail: leserservice@springer.com

P.S. Vieles mehr rund um $\mathrm{hr}$ Fachgebiet finden Sie auf www.springermedizin.de 